\title{
Bioactive constituents of Terminalia ferdinandiana Exell: A pharmacognistic approach towards the prevention and treatment of yersiniosis
}

\author{
Mitchell Henry Wright ${ }^{1}$, Megan Sarah Jean Arnold ${ }^{1,2}$, Huda Aldosary ${ }^{1}$, Joseph Sirdaarta ${ }^{1,3}$, Anthony Carlson Greene ${ }^{1}$, Ian Edwin Cock ${ }^{1,3^{*}}$ \\ 'School of Natural Sciences, Griffith University, 170 Kessels Rd, Nathan, Queensland 4111, AUSTRALIA. \\ 2Eskitis Institute for Drug Discovery, Griffith University,46 Don Young Rd, Nathan, Queensland 4111, AUSTRALIA. \\ Environmental Futures Research Institute, Nathan Campus, Griffith University, 170 Kessels Rd, Nathan, Queensland 4111, AUSTRALIA.
}

\begin{abstract}
Introduction: Yersinia enterocolitica is a facultatively anaerobic gram negative bacterium which contaminates meat products causing the acute gastrointestinal disease yersiniosis. Terminalia ferdinandiana (Kakadu plum, gubinge) is an Australian fruit with an extremely high antioxidant capacity. It was used therapeutically by the first Australians and has documented antiseptic properties against an extensive panel of bacteria. Despite this, it has not been tested for the ability to inhibit the growth of $Y$. enterocolitica. Methods: T. ferdinandiana fruit and leaf extracts were extracted by maceration and the extracts were investigated by disc diffusion assay for growth inhibitory activity against a clinical strain of $Y$. enterocolitica. The MIC values of the extracts were determined to quantify and compare their efficacies. Toxicity was determined using the Artemia franciscana nauplii bioassay. The most potent extracts were investigated using non-targeted GC-MS analysis (with screening against a compound database) for the identification and characterisation of individual components in the crude plant extracts. Results: Solvent extractions of T. ferdinandiana leaf and fruit displayed good growth inhibitory activity in the disc diffusion assay against $Y$. enterocolitica. The methanolic T. ferdinandiana leaf and fruit extracts, as well as the fruit ethyl acetate extract, were particularly potent growth inhibitors, with MIC values of 372,123 and $285 \mu \mathrm{g} / \mathrm{mL}$ respectively. The aqueous and ethyl acetate leaf extracts also displayed good growth inhibitory activity
\end{abstract}

against Y. enterocolitica, albeit with higher MIC values (588 and $1100 \mu \mathrm{g} / \mathrm{mL}$ respectively). All other extracts were either low efficacy, or completely devoid of growth inhibitory activity. All T. ferdinandiana leaf and fruit extracts were either nontoxic $\left(\mathrm{LC}_{50}\right.$ values $<1000 \mu \mathrm{g} / \mathrm{mL}$ ) or of low toxicity in the Artemia franciscana bioassay. Non-biased GC-MS phytochemical analysis of the methanolic extracts putatively identified and highlighted several compounds that may contribute to the ability of these extracts to inhibit the growth of $Y$. enterocolitica. Conclusion: The lack of toxicity and the potent growth inhibitory bioactivity of the $T$. ferdinandiana fruit and leaf methanolic extracts against $Y$. enterocolitica indicates their potential as medicinal agents in the treatment and prevention of yersiniosis.

Key words: Kakadu plum, gubinge, Yersinia enterocolitica, Enterobacteriaceae, antioxidant, antibacterial, terpenoids, GC-MS, metabolomic profile.

\section{Correspondence:}

Ian Edwin Cock, School of Natural Sciences, Griffith University,

170 Kessels Rd, Nathan, Queensland 4111, AUSTRALIA.

Phone no: +61 737357637 ; fax: +61 737355282

E-mail: I.Cock@griffith.edu.au (I. E. Cock)

DOI : 10.5530/pc.2016.3.5

\section{INTRODUCTION}

The genus Yersinia (Y.) comprises a large group of gram-negative, facultatively anaerobic bacteria belonging to the family Enterobacteriaceae. Widespread in the environment and amongst animal populations, the genus is clinically important as some members are pathogenic and are the etiological agents of several diseases in humans. ${ }^{1}$ Pathogenic members of the genus are responsible for a number of fatal and non-fatal infections, ranging from the relatively mild gastrointestinal disease yersiniosis (Y. enterocolitica) to the potentially fatal bubonic plague (Y. pestis). ${ }^{2,3}$ Furthermore, these organisms are zoonotic pathogens and may persist in non-human reservoirs until they are able to infect humans. This poses a unique set of problems otherwise not encountered in the prevention of other diseases, as traditional methods (isolation of infected individuals, targeted immunization) are less effective in the treatment of zoonotic infections. ${ }^{4}$

Yersiniosis is an acute gastrointestinal infection acquired through the ingestion of contaminated food or water. Characterised by abdominal pain, diarrhoea and fever, yersiniosis is a significant disease which accounts for over 100,000 cases of gastroenteritis in the US annually. ${ }^{5}$ The disease originates from several sources although it is a predominately food-borne illness and transmission of this type accounts for approximately $90 \%$ of all reported cases. ${ }^{6}$ Y. enterocolitica has the ability to grow at $4^{\circ} \mathrm{C}$ and thus refrigeration is an inadequate means of protection from infection. ${ }^{7}$ Although rarely fatal and often self-limiting, the symptoms associated with yersiniosis can result in sufferers becoming temporarily incapacitated and thus has indirect negative economic consequences. The probing of natural reservoirs for antibacterial agents effective in the growth inhibition of $Y$. enterocolitica offers an alternative approach in the prevention and treatment of these infections.

Terminalia ferdinandiana Exell (Kakadu plum, gubinge) is an endemic Australian plant which has an extremely high antioxidant content. ${ }^{8,9}$ The fruit of this plant also has the highest ascorbic acid levels of any plant in the world, with levels reported as high as $6 \%$ of the recorded wet weight. ${ }^{8,9}$ This is approximately 900 times higher $(\mathrm{g} / \mathrm{g})$ than the ascorbic acid content in blueberries (which are themselves considered a good source of antioxidants). The medicinal benefits of the fruit were well known by the first Australians who considered the plum both a medicine and a nutritious food source. It has been proposed that many of the health benefits of the fruit may stem from the growth inhibitory activity of its many antimicrobial compounds. ${ }^{10}$ These include flavonoids, benzoic acid derivatives, as well as gallic acid and ellagic acid derivatives, all of which have been previously associated with microbial inhibition. ${ }^{11}$ Indeed, the antiseptic potential of the fruit is well documented in the prevention of several disease-causing microorganisms. ${ }^{12-15}$ Recently, T. ferdinandiana leaf extracts were also shown to have antibacterial activity. ${ }^{16}$ Potent growth inhibitory activity was reported against the bacterial triggers of several auto-immune inflammatory diseases including rheumatoid arthritis 
(Proteus mirabilis), ankylosing spondylitis (Klebsiella pneumoniae) and multiple sclerosis (Acinetobacter baylyi and Pseudomonas aeruginosa). ${ }^{16}$ The study indicated that the inhibition of the bacterial growth by the leaf extracts may be due to their high tannin content. Despite these studies and the extremely high antioxidant capacity of T. ferdinandiana, it is yet to be evaluated for the ability to inhibit the growth of the food poisoning bacterium Y. enterocolitica.

\section{MATERIALS AND METHODS}

\section{Plant source and extraction}

T. ferdinandiana fruit pulp and leaves were provided and verified courtesy of David Boehme of Wild Harvest, Northern Territory (Australia). The pulp was frozen prior to transport and stored at $-10^{\circ} \mathrm{C}$ until processing. A voucher specimen of the pulp (KP2014GD) is maintained at School of Natural Sciences, Griffith University. The pulp and leaves were extensively dehydrated in a Sunbeam food dehydrator and the desiccated material was stored at $-30^{\circ} \mathrm{C}$. A voucher specimen (KP2015LA) is stored at the School of Natural Sciences, Griffith University. The plant materials were comprehensively dried and ground into a coarse powder prior to use. A mass of $1 \mathrm{~g}$ of ground powder was extensively extracted individually by maceration in $50 \mathrm{~mL}$ of deionised water, methanol, hexane, ethyl acetate orchloroform for $24 \mathrm{~h}$ at $4^{\circ} \mathrm{C}$ with gentle shaking. All solvents were supplied by Ajax, Australia and were AR grade. The extracts were filtered through filter paper (Whatman No. 54) and air dried at room temperature. The aqueous extract was lyophilised by rotary evaporation in an Eppendorf concentrator 5301. The subsequent pellets were dissolved in $10 \mathrm{~mL}$ deionised water (containing $0.5 \%$ DMSO). The extract was passed through a $0.22 \mu \mathrm{m}$ filter (Sarstedt) and stored at $4^{\circ} \mathrm{C}$ until used.

\section{Qualitative phytochemical studies}

Phytochemical analysis of the extracts for the presence of tannins, triterpenoids, saponins, flavonoids, phytosteroids, phenolic compounds, anthraquinones, cardiac glycosides and alkaloids were conducted by previously described assays. ${ }^{17-19}$

\section{Antioxidant capacity}

The antioxidant capacity of each sample was assessed using a modified DPPH free radical scavenging method. ${ }^{20-22}$ Ascorbic acid (0-25 $\mu \mathrm{g}$ per well) was used as a reference and the absorbances were measured and recorded at $515 \mathrm{~nm}$. All tests were performed in parallel with controls on each plate and all were performed in triplicate. The antioxidant capacity based on DPPH free radical scavenging ability was determined for each extract and expressed as $\mu$ g ascorbic acid equivalents per gram of original plant material extracted.

\section{Antibacterial screening}

\section{Clinical Yersinia enterocolitica strain}

The clinical isolate strain of Yersinia enterocolitica used in this study was supplied by Ms. Jane Gifkins of the School of Natural Sciences Griffith University, Australia. All growth studies were performed using nutrient agar (Oxoid Ltd., Australia) under aerobic conditions. Incubation was at $30^{\circ} \mathrm{C}$ and the stock culture was subcultured and maintained in nutrient broth at $4^{\circ} \mathrm{C}$.

\section{Evaluation of antimicrobial activity}

Antimicrobial activity of all plant extracts was determined using a modified disc diffusion assay. ${ }^{23-25}$ Briefly, $100 \mu \mathrm{L}$ of $Y$. enterocolitica was grown in $10 \mathrm{~mL}$ of fresh nutrient broth until they reached a count of $\sim 10^{8}$ cells $/ \mathrm{mL}$. A volume of $100 \mu \mathrm{L}$ of the bacterial suspension was spread onto nutrient agar plates and extracts were tested for antibacterial activity using $5 \mathrm{~mm}$ sterilised filter paper discs. Discs were infused with $10 \mu \mathrm{L}$ of T. ferdinandiana extracts, allowed to dry and placed onto the inoculated plates. The plates were allowed to stand at $4^{\circ} \mathrm{C}$ for $2 \mathrm{~h}$ before incubation at $30^{\circ} \mathrm{C}$ for $24 \mathrm{~h}$. The diameters of the inhibition zones were measured to the closest whole millimetre. Each assay was performed in at least triplicate. Mean values $( \pm$ SEM) are reported in this study. Standard discs of chloramphenicol $(10 \mu \mathrm{g})$ were obtained from Oxoid, Australia and were used as positive controls to compare antibacterial activity. Filter discs infused with $10 \mu \mathrm{L}$ of distilled water were used as a negative control.

\section{Minimum inhibitory concentration (MIC) determination}

The minimum inhibitory concentrations (MIC) of the extracts was determined as previously described. ${ }^{26}$ Briefly, the plant extracts were diluted in deionised water and tested across a range of concentrations. Discs were infused with $10 \mu \mathrm{L}$ of the extract dilutions, allowed to dry and placed onto inoculated plates. The assay was performed as outlined above and graphs of the zone of inhibition versus concentration were plotted. Linear regression was used to determine MIC values.

\section{Toxicity screening}

\section{Reference toxin for toxicity screening}

Potassium dichromate $\left(\mathrm{K}_{2} \mathrm{Cr}_{2} \mathrm{O}_{7}\right)$ (AR grade, Chem-Supply, Australia) was prepared in distilled water $(4 \mathrm{mg} / \mathrm{mL})$ and serially diluted in artificial seawater for use in the Artemia franciscana nauplii bioassay.

\section{Artemia franciscana nauplii toxicity screening}

Toxicity was tested using a modified Artemia franciscana nauplii lethality assay. ${ }^{27-29}$ Briefly, $400 \mu \mathrm{L}$ of seawater containing $\sim 43$ (mean $43.2, \mathrm{n}=155$, SD 14.5) A. franciscana nauplii were added to wells of a 48 well plate and immediately used in the bioassay. Volumes of $400 \mu \mathrm{L}$ of the reference toxin or the diluted plant extracts were transferred to the wells and incubated at $25 \pm 1^{\circ} \mathrm{C}$ under artificial light (1000 Lux). A negative control ( $400 \mu \mathrm{L}$ seawater) was run in triplicate for each plate. All treatments were performed in at least triplicate. The wells were checked at regular intervals and the number of dead counted. Mortality was determined if no movement of the appendages was detected within $10 \mathrm{sec}$. After $24 \mathrm{~h}$, all nauplii were sacrificed and counted to determine the total \% mortality per well. The $\mathrm{LC}_{50}$ with $95 \%$ confidence limits for each treatment was calculated using probit analysis.

\section{Non-targeted GC-MS analysis}

Separations were performed with a Shimadzu GC-2010 Plus (USA) linked to a Shimadzu MS TQ8040 (USA) mass spectrometer. The system was equipped with a Shimadzu auto-sampler AOC-5000 Plus (USA). Chromatographic separation was accomplished using a (5\% phenyl, 95\% dimethyl polysiloxane, $30 \mathrm{~m} \times 0.25 \times 0.25 \mathrm{um}$ ) capillary column, obtained from (Restek USA). Helium (99.999\%) was employed as a carrier gas at a flow rate of $0.75 \mathrm{~mL} / \mathrm{min}$ at a split ratio of $21: 1$. The injector temperature was set at $280^{\circ} \mathrm{C}$.

The initial column temperature was held at $40^{\circ} \mathrm{C}$ for $5 \mathrm{~min}$, with an initial ramp at $5^{\circ} \mathrm{C} / \mathrm{min}$ to acquire a temperature of $140^{\circ} \mathrm{C}$ which was subsequently maintained for a further minute. A second ramp was initiated at $20^{\circ} \mathrm{C}$ per minute, to attain a column temperature of $260^{\circ} \mathrm{C}$, and held for a minute. The third ramp of $50^{\circ} \mathrm{C} / \mathrm{min}$ was undertaken to reach a final temperature of $300^{\circ} \mathrm{C}$, which was maintained for a duration of $5 \mathrm{~min}$. The mass spectrometer was operated in the electron ionisation mode at $70 \mathrm{eV}$. The analytes were then recorded in total ion count (TIC) mode. The TIC was acquired after a min and for a duration of $38.8 \mathrm{~min}$ utilising a mass range of $26-500 \mathrm{~m} / \mathrm{z}$. 
Table 1: The mass of dried extracted material, the concentration after resuspension in deionised water, qualitative phytochemical screenings and antioxidant capacities of the $T$. ferdinandiana extracts

\begin{tabular}{|c|c|c|c|c|c|c|c|c|c|c|c|c|c|c|c|c|}
\hline 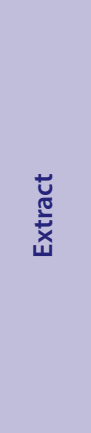 & 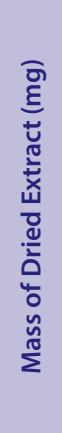 & 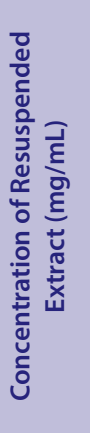 & 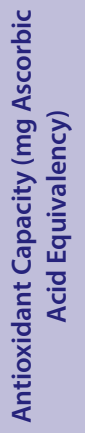 & 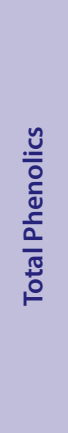 & 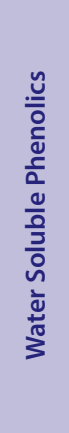 & 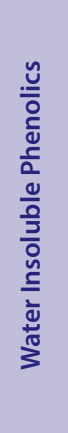 & 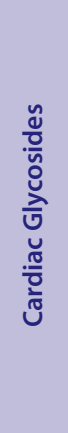 & 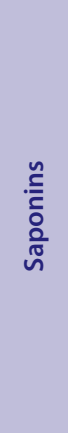 & 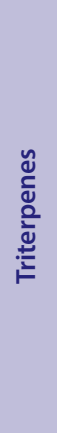 & 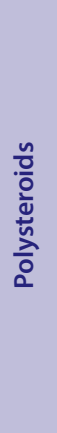 & 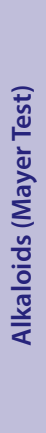 & 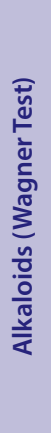 & $\begin{array}{l}\frac{n}{0} \\
\frac{0}{0} \\
\frac{0}{0} \\
\frac{\pi}{4}\end{array}$ & 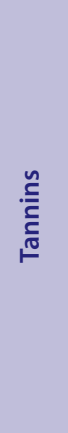 & 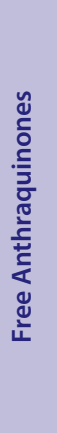 & 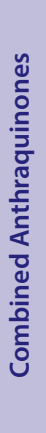 \\
\hline KPLW & 471 & 47.1 & 340 & +++ & +++ & +++ & ++ & +++ & ++ & - & - & - & ++ & +++ & + & + \\
\hline KPLM & 331 & 33.1 & 150 & +++ & +++ & +++ & +++ & ++ & + & - & + & + & ++ & +++ & + & + \\
\hline KPLC & 59 & 5.9 & 5 & + & - & - & - & - & - & - & - & - & - & - & - & - \\
\hline KPLH & 58 & 5.8 & 0.4 & + & - & - & - & - & - & - & - & - & ++ & + & - & - \\
\hline KPLE & 59 & 5.9 & 22 & +++ & +++ & +++ & - & - & - & - & - & - & ++ & ++ & - & - \\
\hline KPFW & 483 & 48.3 & 264 & +++ & +++ & +++ & - & + & - & - & - & - & +++ & ++ & - & - \\
\hline KPFM & 359 & 35.9 & 660 & +++ & +++ & +++ & - & ++ & + & - & + & + & +++ & ++ & - & - \\
\hline KPFC & 62 & 6.2 & 7 & + & - & - & - & - & - & - & - & - & - & - & - & - \\
\hline KPFH & 18 & 1.8 & 1 & - & - & - & - & - & - & - & - & - & - & - & - & - \\
\hline KPFE & 30 & 3 & 39 & ++ & ++ & + & - & + & ++ & - & - & - & ++ & - & - & - \\
\hline
\end{tabular}

+ indicates a minor response; ++ indicates a moderate response; +++ indicates a major response. - indicates no response in the assay. KPLW=aqueous T. ferdinandiana leaf extract; KPLM=methanolic T. ferdinandiana leaf extract; $\mathbf{K P L C}=$ chloroform $T$. ferdinandiana leaf extract; $\mathbf{K P L H}=$ hexane $T$. ferdinandiana leaf

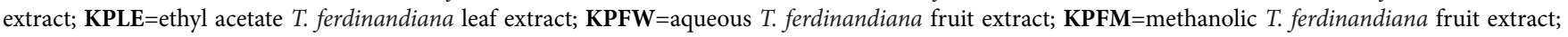

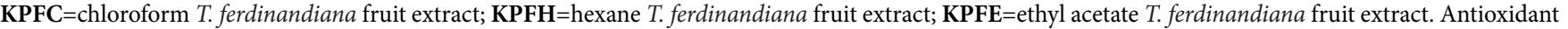
capacity was determined by DPPH reduction and is expressed as mg ascorbic acid equivalents per gof original plant material extracted.

Table 2: Minimum inhibitory concentration $(\mu \mathrm{g} / \mathrm{mL})$ of the $T$. ferdinandiana extracts against $Y$. enterocolitica and $\mathrm{LC}_{50}$ values $(\mu \mathrm{g} / \mathrm{mL})$ in the Artemia nauplii bioassay

\begin{tabular}{ccc}
\hline Extract & MIC & LC $_{50}$ \\
\hline KPLW & 588 & 1,330 \\
KPLM & 372 & 1,133 \\
KPLC & 5900 & - \\
KPLH & 5800 & - \\
KPLE & 1100 & 767 \\
KPFW & - & 2,080 \\
KPFM & 123 & 2,115 \\
KPFC & 6200 & - \\
KPFH & - & - \\
KPFE & 285 & - \\
PD & ND & 186 \\
SW & ND & - \\
\hline
\end{tabular}

Numbers indicate the mean $\mathrm{MIC}$ and $\mathrm{LC}_{50}$ values of triplicate determinations. - indicates no inhibition. $\mathbf{K P L W}=$ aqueous $T$. ferdinandiana leaf extract; $\mathbf{K P L M}=$ methanolic $T$. ferdinandiana leaf extract; $\mathbf{K P L C}=$ chloroform $T$. ferdinandiana leaf extract; $\mathbf{K P L H}=$ hexane $T$. ferdinandiana leaf extract; $\mathbf{K P L E}=$ ethyl acetate $T$. ferdinandiana leaf extract; $\mathbf{K P F W}=$ aqueous $T$. ferdinandiana fruit extract; $\mathbf{K P F M = m e t h a n o l i c ~} T$. ferdinandiana fruit extract; $\mathbf{K P F C}=$ chloroform T. ferdinandiana fruit extract; $\mathbf{K P F H}=$ hexane T. ferdinandiana fruit extract; $\mathbf{K P F E}=$ ethyl acetate $T$. ferdinandiana fruit extract; $\mathbf{P D}=$ potassium dichromate control; $\mathbf{S W}=$ artificial seawater negative control; $\mathrm{ND}=$ the indicated test was not performed. 
Table 3: Qualitative GC-MS analysis of methanolic T. ferdinandiana fruit extract, elucidation of empirical formulas and putative identification of each compound

\begin{tabular}{|c|c|c|c|c|}
\hline \multicolumn{5}{|c|}{ T. ferdinandiana fruit methanolic extract } \\
\hline Molecular Mass & Molecular Formula & Putative Identification & $\begin{array}{l}\text { Retention } \\
\text { Time }\end{array}$ & Area\% \\
\hline 110 & $\mathrm{C}_{5} \mathrm{H}_{6} \mathrm{~N}_{2} \mathrm{O}$ & 1-(3H-Imidazol-4-yl)-ethanone & 10.216 & 30.22 \\
\hline 118 & $\mathrm{C}_{6} \mathrm{H}_{14} \mathrm{O}_{2}$ & 3-Methoxy-3-methylbutanol & 10.63 & 0.04 \\
\hline 132 & $\mathrm{C}_{5} \mathrm{H}_{8} \mathrm{O}_{4}$ & Propanedioic acid, dimethyl ester & 10.859 & 0.79 \\
\hline 128 & $\mathrm{C}_{8} \mathrm{H}_{16} \mathrm{O}$ & 4-methyl-2-heptanone & 11.191 & 1.26 \\
\hline 106 & $\mathrm{C}_{7} \mathrm{H}_{6} \mathrm{O}$ & Benzaldehyde & 11.755 & 3.23 \\
\hline 126 & $\mathrm{C}_{6} \mathrm{H}_{6} \mathrm{O}_{3}$ & Methyl 2-furoate & 12.325 & 10.07 \\
\hline 128 & $\mathrm{C}_{8} \mathrm{H}_{16} \mathrm{O}$ & Octanal & 13.24 & 0.26 \\
\hline 278 & $\mathrm{C}_{14} \mathrm{H}_{30} \mathrm{O}_{3} \mathrm{~S}$ & Sulfurous acid, 2-ethylhexyl hexyl ester & 13.705 & 0.15 \\
\hline 142 & $\mathrm{C}_{10} \mathrm{H} 22$ & 3,3,5-Trimethylheptane & 13.92 & 0.59 \\
\hline 130 & $\mathrm{C}_{8} \mathrm{H}_{18} \mathrm{O}$ & 2-Ethylhexanol & 14.105 & 4.81 \\
\hline 146 & $\mathrm{C}_{6} \mathrm{H}_{10} \mathrm{O} 4$ & Butanedioic acid, dimethyl ester & 14.205 & 3.4 \\
\hline 120 & $\mathrm{C}_{8} \mathrm{H}_{8} \mathrm{O}$ & Benzeneacetaldehyde & 14.425 & 0.44 \\
\hline 298 & $\mathrm{C}_{19} \mathrm{H}_{38} \mathrm{O}_{2}$ & Oxirane, [(hexadecyloxy)methyl]- & 14.85 & 0.06 \\
\hline 168 & $\mathrm{C}_{12} \mathrm{H}_{24}$ & 4-methyl-1-undecene & 15.08 & 0.58 \\
\hline 160 & $\mathrm{C}_{7} \mathrm{H}_{12} \mathrm{O}_{4}$ & Butanedioic acid, methyl-, dimethyl ester & 15.265 & 0.54 \\
\hline 170 & $\mathrm{C}_{10} \mathrm{H}_{18} \mathrm{O}_{2}$ & cis-Linalool oxide & 15.475 & 3.41 \\
\hline 120 & $\mathrm{C}_{8} \mathrm{H}_{8} \mathrm{O}$ & Bicyclo[4.2.0]octa-1,3,5-trien-7-ol & 15.62 & 0.25 \\
\hline 126 & $\mathrm{C}_{6} \mathrm{H}_{6} \mathrm{O}_{3}$ & Furylhydroxymethyl ketone & 15.685 & 1.1 \\
\hline 170 & $\mathrm{C}_{10} \mathrm{H}_{18} \mathrm{O}_{2}$ & trans-Linalool oxide & 15.96 & 2.1 \\
\hline 136 & $\mathrm{C}_{7} \mathrm{H}_{8} \mathrm{~N}_{2} \mathrm{O}$ & Benzhydrazide & 16.08 & 0.53 \\
\hline 142 & $\mathrm{C}_{9} \mathrm{H}_{18} \mathrm{O}$ & Nonanal & 16.45 & 0.35 \\
\hline 162 & $\mathrm{C}_{6} \mathrm{H}_{10} \mathrm{O}_{5}$ & Dimethyl dl-malate & 17.09 & 0.43 \\
\hline 160 & $\mathrm{C}_{7} \mathrm{H}_{12} \mathrm{O}_{4}$ & Pentanedioic acid, dimethyl ester & 17.445 & 0.2 \\
\hline 110 & $\mathrm{C}_{6} \mathrm{H}_{6} \mathrm{O}_{2}$ & Ethanone, 1-(2-furanyl)- & 17.485 & 0.22 \\
\hline 152 & $\mathrm{C}_{10} \mathrm{H}_{16} \mathrm{O}_{2}$ & Camphor & 17.645 & 0.04 \\
\hline 111 & $\mathrm{C}_{4} \mathrm{H}_{5} \mathrm{~N}_{3} \mathrm{O}$ & 2-(Cyanoimino)oxazolidine & 17.89 & 4.19 \\
\hline 154 & $\mathrm{C}_{10} \mathrm{H}_{18} \mathrm{O}$ & endo-Borneol & 18.32 & 1.3 \\
\hline 144 & $\mathrm{C}_{8} \mathrm{H}_{16} \mathrm{O}_{2}$ & Octanoic acid & 18.56 & 0.39 \\
\hline 150 & $\mathrm{C}_{9} \mathrm{H}_{10} \mathrm{O}_{2}$ & Benzeneacetic acid, methyl ester & 18.61 & 0.27 \\
\hline 158 & $\mathrm{C}_{9} \mathrm{H}_{18} \mathrm{O}_{2}$ & (E)-1-(1-methoxyethoxy)-3-hexene & 18.925 & 0.05 \\
\hline 184 & $\mathrm{C}_{13} \mathrm{H}_{28}$ & 5-(2-methylpropyl)-nonane & 19.39 & 0.18 \\
\hline 134 & $\mathrm{C}_{9} \mathrm{H}_{10} \mathrm{O}$ & 2-methyl-2-phenyl-oxirane & 19.7 & 3.4 \\
\hline 186 & $\mathrm{C}_{10} \mathrm{H}_{18} \mathrm{O}_{3}$ & 2,2-Dimethylpropanoic anhydride & 19.86 & 0.12 \\
\hline 126 & $\mathrm{C}_{6} \mathrm{H}_{6} \mathrm{O}_{3}$ & 5-Hydroxymethylfurfural & 19.945 & 0.42 \\
\hline 184 & $\mathrm{C}_{13} \mathrm{H}_{28}$ & 4-methyl-dodecane & 20.035 & 0.06 \\
\hline 268 & $\mathrm{C}_{19} \mathrm{H}_{40}$ & 5-methyl-octadecane & 20.675 & 0.05 \\
\hline 140 & $\mathrm{C}_{9} \mathrm{H}_{16} \mathrm{O}$ & 2,3,4,5-Tetramethylcyclopent-2-en-1-ol & 20.78 & 0.16 \\
\hline 190 & $\mathrm{C}_{14} \mathrm{H}_{22} \mathrm{O}$ & Benzene, 1,3-bis(1,1-dimethylethyl)- & 20.93 & 2.56 \\
\hline 158 & $\mathrm{C}_{9} \mathrm{H}_{18} \mathrm{O}_{2}$ & Nonanoic acid & 21.31 & 1.02 \\
\hline 168 & $\mathrm{C}_{12} \mathrm{H}_{24}$ & 4-methyl-1-undecene & 21.705 & 0.23 \\
\hline 188 & $\mathrm{C}_{10} \mathrm{H}_{20} \mathrm{O}_{3}$ & Butyl 2-butoxyacetate & 21.84 & 0.31 \\
\hline 114 & $\mathrm{C}_{8} \mathrm{H}_{18}$ & 3,3-dimethyl-hexane & 22.96 & 0.04 \\
\hline 180 & $\mathrm{C}_{13} \mathrm{H}_{24}$ & 1,1,6,6-Tetramethylspiro[4.4]nonane & 23.35 & 0.36 \\
\hline 286 & $\mathrm{C}_{16} \mathrm{H}_{30} \mathrm{O}_{4}$ & 2,2,4-Trimethyl-1,3-pentanediol diisobutyrate & 23.615 & 0.12 \\
\hline
\end{tabular}


Table 3: Continued

\begin{tabular}{lcccc}
$\mathbf{1 7 2}$ & $\mathrm{C}_{10} \mathrm{H}_{20} \mathrm{O}_{2}$ & n-Decanoic acid & 23.87 & 0.03 \\
$\mathbf{2 1 6}$ & $\mathrm{C}_{12} \mathrm{H}_{24} \mathrm{O}_{3}$ & Propanoic acid, 2-methyl-, 3-hydroxy-2,2,4-trimethylpentyl ester & 24.17 & 0.17 \\
$\mathbf{2 2 6}$ & $\mathrm{C}_{14} \mathrm{H}_{26} \mathrm{O}_{2}$ & 2,4,7,9-Tetramethyl-5-decyn-4,7-diol & 25.39 & 25.49 \\
$\mathbf{1 5 0}$ & $\mathrm{C}_{11} \mathrm{H}_{18}$ & Bicyclo[2.2.1]heptane, 2-(1-buten-3-yl)- & 26.29 & 0.12 \\
$\mathbf{2 0 1}$ & $\mathrm{C}_{10} \mathrm{H}_{18} \mathrm{O}_{4}$ & Suberic acid, dimethyl ester & 27.415 & 0.03 \\
$\mathbf{1 8 6}$ & $\mathrm{C}_{12} \mathrm{H}_{26} \mathrm{O}$ & 1-Dodecanol & 28.86 & 0.12 \\
$\mathbf{2 0 6}$ & $\mathrm{C}_{14} \mathrm{H}_{22} \mathrm{O}$ & 3,5-bis(1,1-dimethylethyl)-Phenol & 29.375 & 0.06 \\
$\mathbf{1 6 6}$ & $\mathrm{C}_{9} \mathrm{H}_{10} \mathrm{O}_{3}$ & Ethyl 3-hydroxybenzoate & 30.22 & 0.02 \\
$\mathbf{2 1 6}$ & $\mathrm{C}_{11} \mathrm{H}_{20} \mathrm{O}_{4}$ & Nonanedioic acid, dimethyl ester & 31.77 & 0.18 \\
$\mathbf{2 8 6}$ & $\mathrm{C}_{16} \mathrm{H}_{30} \mathrm{O}_{4}$ & 2,2,4-Trimethyl-1,3-pentanediol diisobuty & 35.92 & 0.02 \\
$\mathbf{3 6 8}$ & $\mathrm{C}_{24} \mathrm{H}_{48} \mathrm{O}_{2}$ & Heneicosanoic acid, isopropyl ester & 36.475 \\
$\mathbf{2 7 8}$ & $\mathrm{C}_{16} \mathrm{H}_{22} \mathrm{O}_{4}$ & Phthalic acid, diisobutylo ester & 0.03 \\
$\mathbf{2 7 8}$ & $\mathrm{C}_{16} \mathrm{H}_{22} \mathrm{O}_{4}$ & 2-(1-oxopropyl)-benzoic acid & 37.62 \\
\hline
\end{tabular}

The relative abundance is a measure of the area under the peak expressed as a $\%$ of the total area under all chromatographic peaks.

Table 4: Qualitative GC-MS analysis of methanolic T. ferdinandiana leaf extract, elucidation of empirical formulas and putative identification of each compound

\begin{tabular}{ccccc}
\hline & & T. ferdinandiana leaf methanolic extract & \\
\hline Molecular Mass & Molecular Formula & Putative Identification & Retention Time & Area\% \\
\hline $\mathbf{1 5 1}$ & $\mathrm{C}_{8} \mathrm{H}_{9} \mathrm{NO}_{2}$ & Methyoxy-phenyl-oxime & 11.266 & 19.7 \\
$\mathbf{1 2 8}$ & $\mathrm{C}_{8} \mathrm{H}_{16} \mathrm{O}$ & 1-Octen-3-ol & 13.727 & 6.35 \\
$\mathbf{1 1 8}$ & $\mathrm{C}_{6} \mathrm{H}_{14} \mathrm{O}_{2}$ & 14.505 & 1.26 \\
$\mathbf{1 3 0}$ & $\mathrm{C}_{8} \mathrm{H}_{18} \mathrm{O}$ & Ethanol, 2-(1,1-dimethylethoxy)- & 15.373 & 0.59 \\
$\mathbf{1 5 4}$ & $\mathrm{C}_{10} \mathrm{H}_{18} \mathrm{O}$ & 1-Hexanol, 2-ethyl- & 15.499 & 0.97 \\
$\mathbf{2 4 2}$ & $\mathrm{C}_{13} \mathrm{H}_{22} \mathrm{O}_{4}$ & Ethyl 2-(5-methyl-5-vinyltetrahydrofuran-2 (linalool oxide) & 16.879 & 0.56 \\
$\mathbf{1 4 2}$ & $\mathrm{C}_{9} \mathrm{H}_{18} \mathrm{O}$ & Nonanal & 17.873 & 1.11 \\
$\mathbf{1 5 0}$ & $\mathrm{C}_{10} \mathrm{H}_{14} \mathrm{O}$ & 21.031 & 0.25 \\
$\mathbf{1 5 6}$ & $\mathrm{C}_{10} \mathrm{H}_{20} \mathrm{O}$ & 2-Isopropylidene-3-methylhexa-3,5-dienal & 21.11 & 0.14 \\
$\mathbf{1 3 4}$ & $\mathrm{C}_{9} \mathrm{H}_{10} \mathrm{O}$ & Decanal & 21.527 & 8.21 \\
$\mathbf{2 1 6}$ & $\mathrm{C}_{12} \mathrm{H}_{24} \mathrm{O}_{3}$ & Benzaldehyde, 3,5-dimethyl- & 26.499 & 2.5 \\
$\mathbf{2 0 6}$ & $\mathrm{C}_{14} \mathrm{H}_{22} \mathrm{O}$ & Propanoic acid, 2-methyl-, 3-hydroxy-2,2,4 & 31.641 & 0.54 \\
$\mathbf{1 9 4}$ & $\mathrm{C}_{11} \mathrm{H}_{14} \mathrm{O}_{3}$ & 2,4-Di-tert-butylphenol & 32.054 & 3.16 \\
$\mathbf{2 8 6}$ & $\mathrm{C}_{16} \mathrm{H}_{30} \mathrm{O}_{4}$ & Ethyl para-ethoxybenzoate & 33.822 & 0.4 \\
\hline
\end{tabular}

The relative abundance is a measure of the area under the peak expressed as a \% of the total area under all chromatographic peaks.

\section{Statistical analysis}

Data is expressed as the mean \pm SEM of at least three independent experiments.

\section{RESULTS}

\section{Liquid extraction yields and qualitative phytochemical screening}

T. ferdinandiana solvent extractions yielded dried plant extracts ranging from $58 \mathrm{mg}$ to $471 \mathrm{mg}$ (leaf extracts) and from $18 \mathrm{mg}$ to $483 \mathrm{mg}$ (fruit extracts) (Table 1). Methanolic and aqueous extracts provided considerably greater yields of extracted material relative to the ethyl acetate, chloroform and hexane counterparts, which gave low to moderate yields. The dried extracts were resuspended in $10 \mathrm{~mL}$ of deionised water (containing 1\% DMSO), resulting in the concentrations presented in Table 1.

\section{Antioxidant content}

Antioxidant capacity for the plant extracts (Table 1) ranged from $0.4 \mathrm{mg}$ (hexane leaf extract) to a high of $660 \mathrm{mg}$ (methanolic fruit extract) ascorbic acid equivalence per gram of dried plant material extracted. The methanolic and aqueous extracts typically had higher antioxidant capacities than the corresponding ethyl acetate, hexane and chloroform extracts.

\section{Antimicrobial activity}

To determine the ability of the T. ferdinandiana fruit and leaf extracts to inhibit $Y$. enterocolitica growth, $10 \mu \mathrm{L}$ of each extract was screened using a disc diffusion assay. Bacterial growth was strongly inhibited by 8 of the 10 extracts screened (80\%) (Figure 1). The aqueous and methanolic leaf extracts were the most potent inhibitors of growth (as judged by zone of inhibition), with inhibition zones of $18 \pm 0.6$ and $17.3 \pm 1.5 \mathrm{~mm}$ respectively. This compares positively with the chloramphenicol control $(10 \mu \mathrm{g}$; 
inhibition zone $=9 \pm 0.6 \mathrm{~mm})$. The methanolic fruit extract also displayed good inhibition of Y. enterocolitica growth, with a zone of inhibition $\geq 15 \mathrm{~mm}$. Typically, the leaf extracts were more potent inhibitors of Y. enterocolitica growth than the corresponding fruit extracts (as judged by inhibition zone).

The antimicrobial efficacy was further quantified through the determination of MIC values against the T. ferdinandiana extracts (Table 2). Most of the extracts were effective at inhibiting $Y$. enterocolitica growth with MIC values $<1000 \mu \mathrm{g} / \mathrm{mL}$ determined for several of the extracts $(<10 \mu \mathrm{g}$ impregnated in the disc). The methanolic extracts of both the leaf and fruit were particularly potent, with MIC values of $372 \mu \mathrm{g} / \mathrm{mL}$ (approximately $3.7 \mu \mathrm{g}$ infused into the disc) and $123 \mu \mathrm{g} / \mathrm{mL}$ (approximately $1.2 \mu \mathrm{g}$ infused into the disc) respectively. These results compare well with the growth inhibitory activity of the chloramphenicol controls which were tested at $10 \mu$ gper disc. The aqueous and ethyl acetate leaf extracts, as well as the fruit ethyl acetate extract, were also potent $Y$. enterocolitica growth inhibitors (MIC values of 588, 1100 and $285 \mu \mathrm{g} / \mathrm{mL}$ respectively). Whilst less potent, the leaf chloroform $(\mathrm{MIC}=5900 \mu \mathrm{g} / \mathrm{mL})$ and hexane extracts $(\mathrm{MIC}=5800 \mu \mathrm{g} / \mathrm{mL})$, as well as the fruit chloroform extract $(\mathrm{MIC}=6200 \mu \mathrm{g} / \mathrm{mL})$, also inhibited $Y$. enterocolitica growth. Their MIC values indicate moderate to low inhibitory activity.

\section{Quantification of toxicity}

All extracts were initially screened in the assay at $2000 \mu \mathrm{g} / \mathrm{mL}$ (Figure 2). As a reference toxin, potassium dichromate was also tested in the bioassay. The reference toxin was prompt in its onset of mortality, inducing nauplii death within the first $3 \mathrm{~h}$ of exposure and $100 \%$ mortality was evident within 4-5 h (unpublished results). All methanolic, aqueous extracts and ethyl acetate leaf extracts showed $>90 \%$ mortality rates at $24 \mathrm{~h}$. All other extracts exhibited $<10 \%$ mortality rates at $24 \mathrm{~h}$ with the exception of the chloroform leaf extract.

To further assess the effects of toxin concentration on the initiation of mortality, the extracts were serially diluted in artificial seawater to test across a series of concentrations in the Artemia nauplii bioassay. The $\mathrm{LC}_{50}$ values of the $T$. ferdinandiana extracts towards $A$. franciscana following $24 \mathrm{~h}$ exposure are displayed in Table 2. No $\mathrm{LC}_{50}$ values are reported for the chloroform or hexane extracts, nor for the ethyl acetate fruit extract as $<50 \%$ mortality was seen in all tested concentrations. Extracts with an $\mathrm{LC}_{50}$ greater than $1000 \mu \mathrm{g} / \mathrm{mL}$ towards Artemia nauplii have previously been defined as nontoxic in this assay. ${ }^{30}$ As only the ethyl acetate fruit extract had an $\mathrm{LC}_{50}$ value of $<1000 \mu \mathrm{g} / \mathrm{ml}$, all other extracts were considered nontoxic. Whilst the $\mathrm{LC}_{50}$ value for the ethyl acetate leaf extract is $<1000 \mu \mathrm{g} / \mathrm{mL}$, a value of $767 \mu \mathrm{g} / \mathrm{mL}$ is representative of low to moderate toxicity.

\section{GC-MS head space analysis}

As the T. ferdinandiana leaf and fruit methanolic extracts were the most potent inhibitor of Y. enterocolitica (as judged by MIC), they were considered the most promising extracts for further phytochemical analysis. Optimised GC-MS parameters were developed and used to examine these extracts. Numerous overlapping peaks were evident in the methanolic fruit extract chromatogram (Figure 3). A total of 57 peaks were detected and putatively identified in that extract, with prominent peaks eluting at $10.2 \mathrm{~min}$ (1-(3H-imidazol-4-yl)-ethanone), $12.3 \mathrm{~min}$ (methyl2-furoate), $14.1 \mathrm{~min}$ (2-ethylhexanol), $15.5 \mathrm{~min}$ (cis-linalool oxide), 16.0 min (trans-linalool oxide), $17.9 \mathrm{~min}$ (2-(cyanoimino) oxazolidine, 19.7 min (2-methyl-2-phenyl-oxirane), 20.9 min (1,3-bis(1,1-dimethylethyl)benzene) and $28.9 \mathrm{~min}$ (3,5-bis(1,1-dimethylethyl)-phenol). Numerous overlapping peaks were also evident throughout the chromatogram, with a broad range of retention times, particularly between 10 and $25 \mathrm{~min}$, attesting to the wide range of compounds of widely varying polarities in the methanolic fruit extract.
The T. ferdinandiana methanolic leaf extract GC-MS chromatogram (Figure 4) was substantially less complex than the corresponding fruit extract chromatogram. Indeed, only 15 peaks were detected and putatively identified in this chromatogram. Major peaks were evident at $11.3 \mathrm{~min}$ (methoxy-phenyl-oxime), $13.8 \mathrm{~min}$ (1-octen-3-ol), $14.4 \mathrm{~min}$ (2-(1,1-dimethylethoxy)-ethanol, $21.5 \mathrm{~min}$ (3,5-dimethyl-benzaldehyde) and 26.5 min (2-methyl-3-hydroxy-2,2,4-propanoic acid). Further prominent peaks were present at 17.0, 18.2, 19.6 and $24.8 \mathrm{~min}$. However, the compound database used in our studies was unable to putatively identify these compounds. Table 4 lists only the compounds which were putatively identified by comparison with the compound database.

\section{DISCUSSION}

Many Terminalia spp. have a history of therapeutic usage to treat microbial infections and numerous recent investigations have reported on their antibacterial properties. ${ }^{31}$ The Australian species T. ferdinandiana has proven to be particularly effective, with growth inhibitory activity reported against a broad panel of bacterial pathogens, ${ }^{32}$ including several bacteria associated with diarrhoea and dysentery. ${ }^{32,33}$ T. ferdinandiana extracts also inhibit the growth of bacterial triggers of rheumatoid arthritis ${ }^{14,16}$ and multiple sclerosis. ${ }^{13}$ Furthermore, T. ferdinandiana has also recently been reported to inhibit the proliferation of the gastrointestinal protozoan parasite Giardia duodenalis, ${ }^{11}$ indicating its therapeutic potential against both prokaryotic and eukaryotic pathogens. However, despite the wealth of information into the therapeutic potential of T. ferdinandiana, it is yet to be comprehensively studied for antibacterial activity against many bacterial species associated with food poisoning. Here we report, growth inhibitory activity for T. ferdinandiana extracts against the bacterial cause of the gastrointestinal disease yersiniosis, an acute infection acquired through the ingestion of food (particularly pork $)^{34}$ contaminated with $Y$. enterocolitica bacteria.

Potent Y. enterocolitica growth inhibitory activity was evident in the T. ferdinandiana fruit and leaf extracts. The methanolic extracts were the most potent growth inhibitors, with MIC values of 123 and $372 \mu \mathrm{g} / \mathrm{mL}$ for the fruit and leaf extracts respectively. Qualitative GC-MS analysis of these extracts identified a number of interesting compounds which may contribute to this activity. The presence of the furan compounds methyl 2-furoate (Figure 5c), linalool oxide (Figure 5e), furylhydroxymethyl ketone (Figure 5g),1-(2-furanyl)-ethanone (Figure 5i) 5-hydroxymethylfurfural (Figure 5o) in the methanolic T. ferdinandiana extracts is particularly noteworthy as many furan derivatives are potent inhibitors of bacterial growth. The nitro furans have particularly well studied antimicrobial mechanisms, acting via the inhibition of nucleic acid synthesis..$^{35}$ Thus, the furan compounds in this extract may have cytostatic $Y$. enterocolitica growth inhibitory mechanisms although this is yet to be investigated. Further studies reported synthetic furan derivatives (modified by the addition of a rhodanine moiety) to be potent inhibitors of the growth of a panel of multidrug resistant bacteria, with MIC values as low as $2 \mu \mathrm{g} / \mathrm{mL}$ against some species. ${ }^{36}$ Whilst we were unable to find reports of anti-bacterial activity for the furan derivatives present in the T. ferdinandiana extracts, it is possible that they may contribute to the growth inhibitory activities reported in our study.

Also noteworthy, an imidazole compound,1-(3H-imidazol-4-yl)-ethanone (Figure 5a), was putatively identified in the methanolic T. ferdinandiana fruit extract. Several imidazo-compounds (e.g. metronidazole, azanidazole tinidazole) have notable antibiotic activity. ${ }^{37}$ Interestingly, similar imidazole compounds also have inhibitory activity against eukaryotic parasites. ${ }^{40}$ Indeed, anti-giardial activity has been reported for T. ferdinandiana fruit extract. ${ }^{11}$ A variety of benzene and benzoic acid derivatives including benzaldehyde (Figure 5b), benzene acetaldehyde (Figure 5d), bicyclo[4.2.0] octa-1,3,5-trien-7-ol (Figure 5f), benzhydrazide 


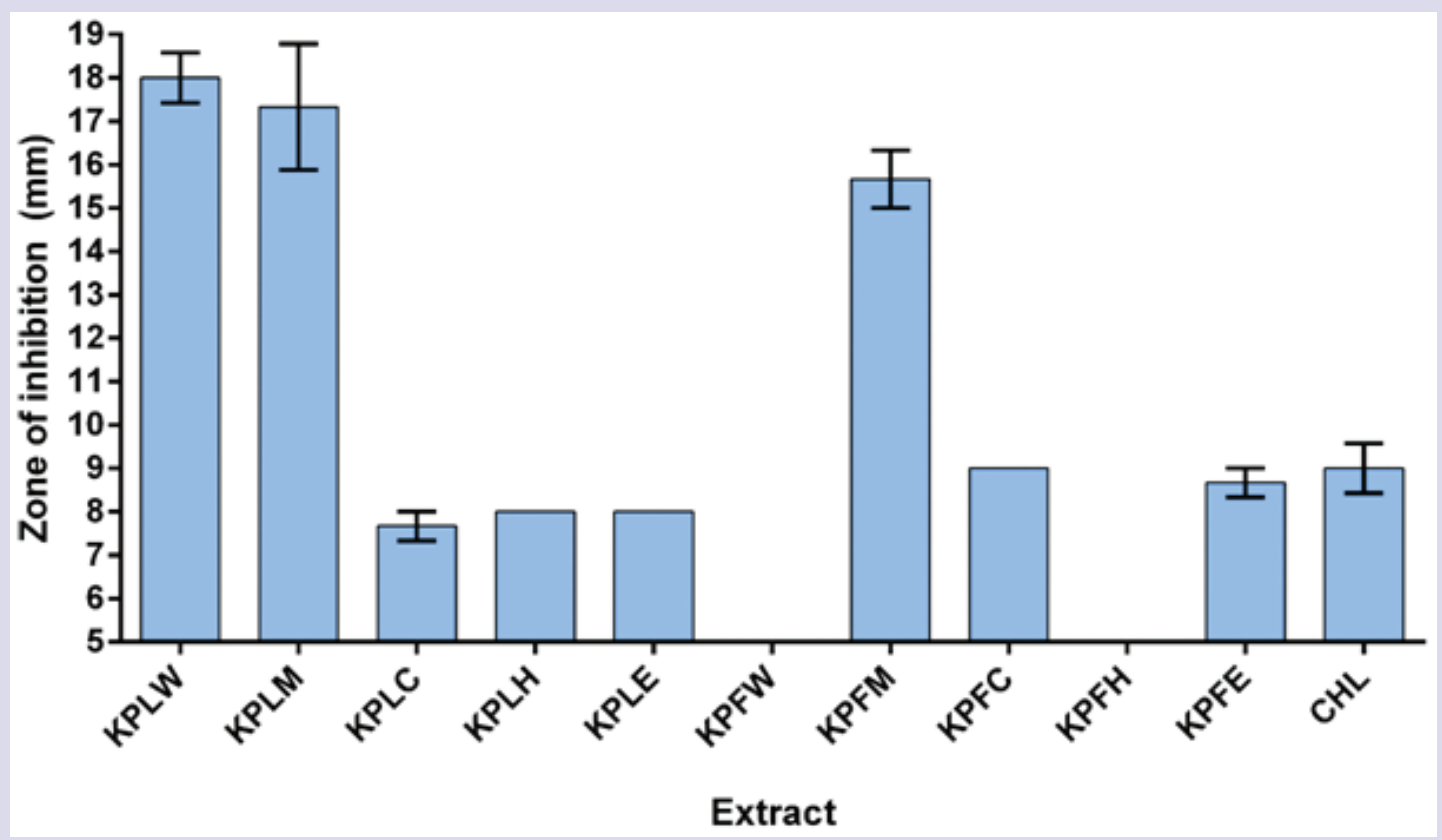

Figure 1: Growth inhibitory activity of $T$. ferdinandianaplant extracts against the $Y$. enterocolitica environmental isolate measured as zones of inhibition (mm). $\mathbf{K P L W}=$ aqueous $T$. ferdinandiana leaf extract; $\mathbf{K P L M}=$ methanolic $T$. ferdinandiana leaf extract; $\mathbf{K P L C}=\mathrm{chloroform} T$. ferdinandiana leaf extract; $\mathbf{K P L H}=$ hexane $T$. ferdinandiana leaf extract; $\mathbf{K P L E}=$ ethyl acetate $T$. ferdinandiana leaf extract; $\mathbf{K P F W = a q u e o u s ~} T$. ferdinandiana fruit extract; $\mathbf{K P F M = m e t h a n o l i c ~} T$. ferdinandiana fruit extract; $\mathbf{K P F C}=$ chloroform $T$. ferdinandiana fruit extract; $\mathbf{K P F H}=$ hexane $T$. ferdinandiana fruit extract; $\mathbf{K P F E = e t h y l ~ a c e t a t e ~} T$. ferdinandiana fruit extract; $\mathrm{CHL}=$ chloramphenicol $(10 \mu \mathrm{g})$. Results are expressed as mean zones of inhibition $\pm \mathrm{SEM}$.

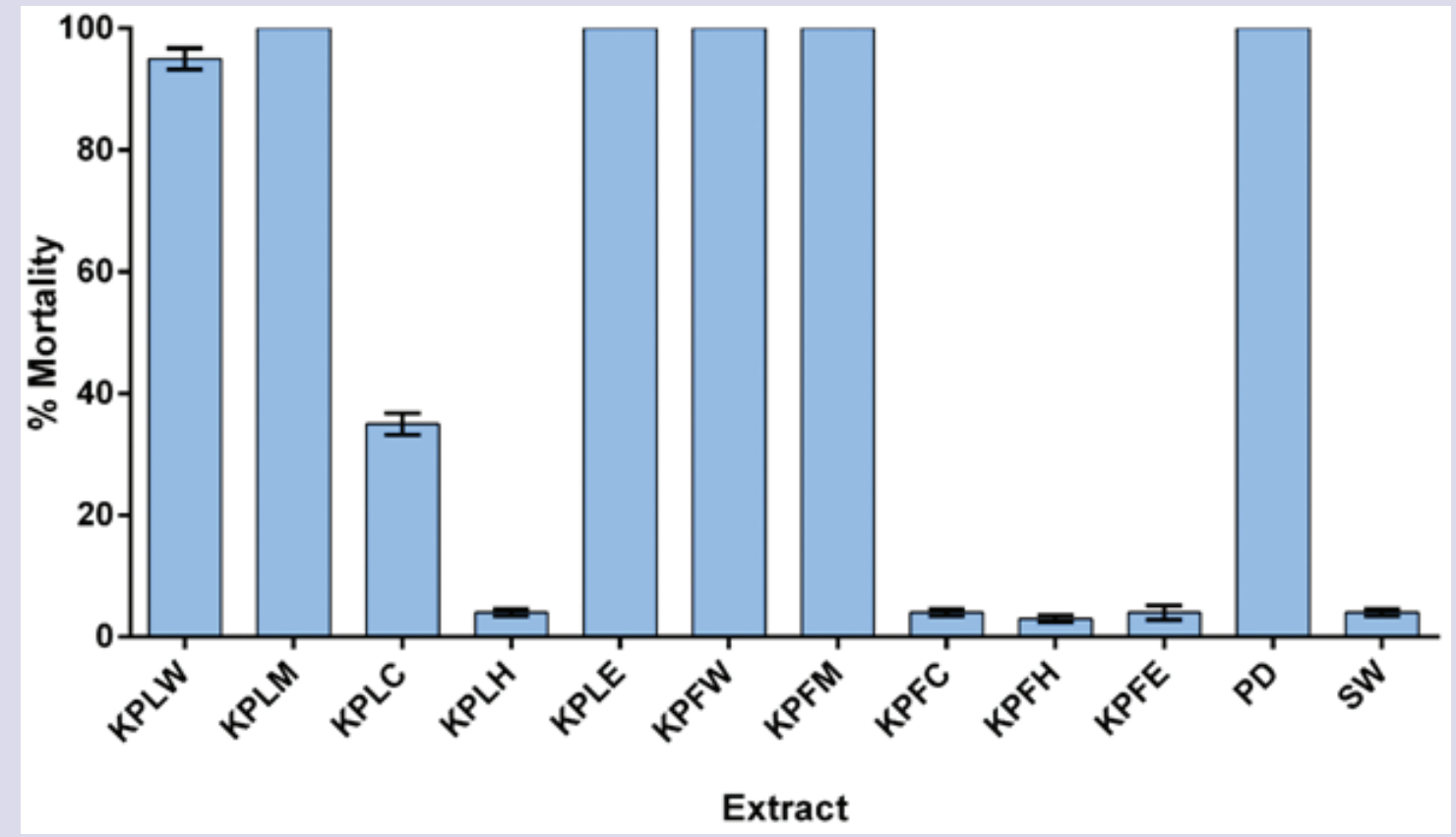

Figure 2: The lethality of the T. ferdinandiana extracts $(2000 \mu \mathrm{g} / \mathrm{mL})$ and the potassium dichromate control $(1000 \mu \mathrm{g} / \mathrm{mL})$ towards Artemia franciscana nauplii after $24 \mathrm{~h}$ exposure. $\mathbf{K P L W}=$ aqueous T. ferdinandiana leaf extract; $\mathbf{K P L M}=$ methanolic $T$. ferdinandiana leaf extract; $\mathbf{K P L C}=$ chloroform . ferdinandiana leaf extract; $\mathbf{K P L H}=$ hexane $T$. ferdinandiana leaf extract; $\mathbf{K P L E}=$ ethyl acetate $T$. ferdinandiana leaf extract; $\mathbf{K P F W}=$ aqueous $T$. ferdinandiana fruit extract; $\mathbf{K P F} \mathbf{M}=$ methanolic T. ferdinandiana fruit extract; $\mathbf{K P F C}=$ chloroform $T$. ferdinandiana fruit extract; $\mathbf{K P F H}=$ hexane $T$. ferdinandiana fruit extract; $\mathbf{K P F E}=\mathrm{ethyl}$ acetate $T$. ferdinandiana fruit extract; PD=potassium dichromate control; SW=seawater control. Results are expressed as mean \% mortality \pm SEM. 


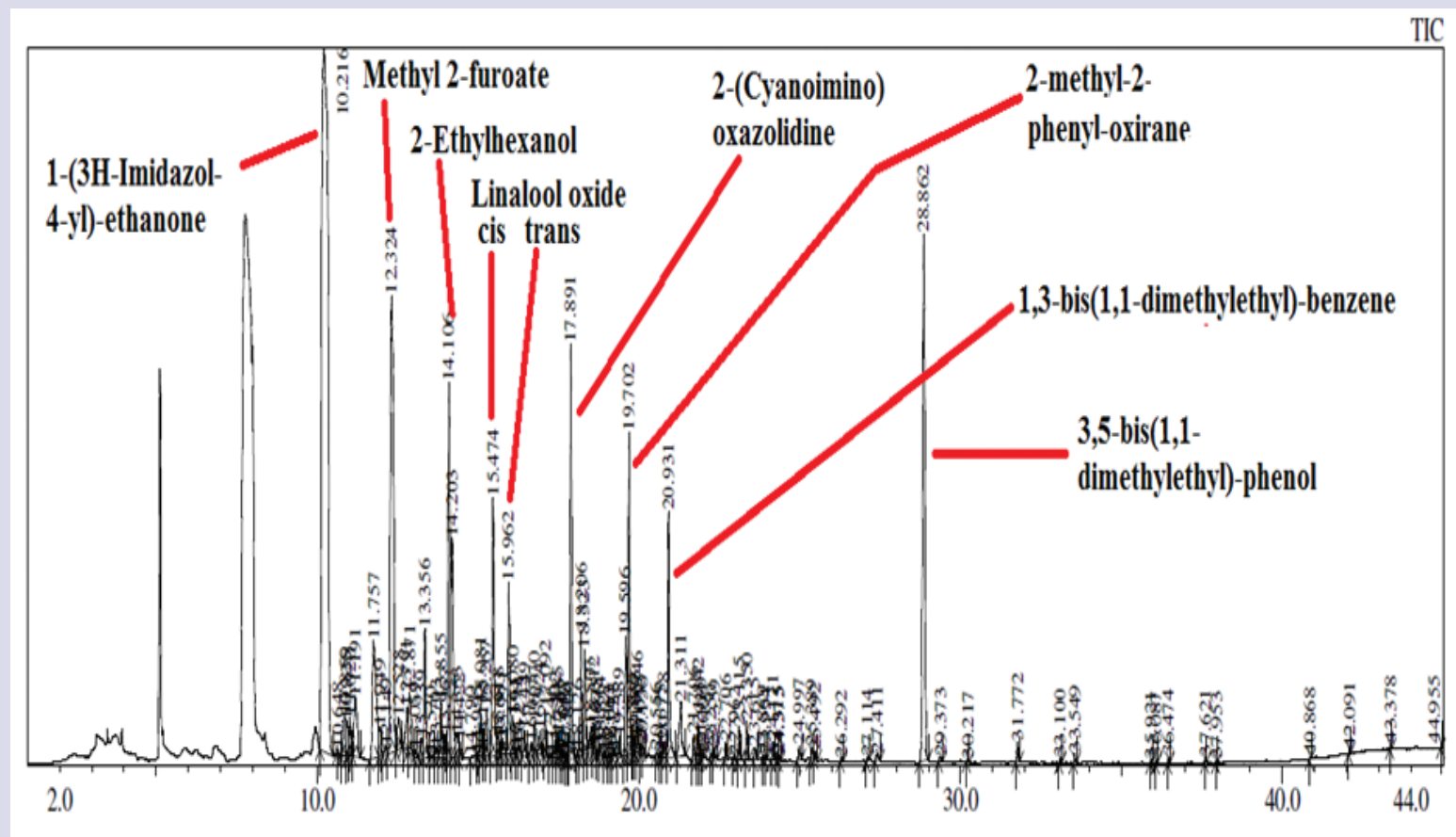

Figure 3: Gas chromatogram of a $0.5 \mu \mathrm{L}$ injection of T. ferdinandiana methanolic fruit extract. The extract was dried and resuspended in methanol for analysis.

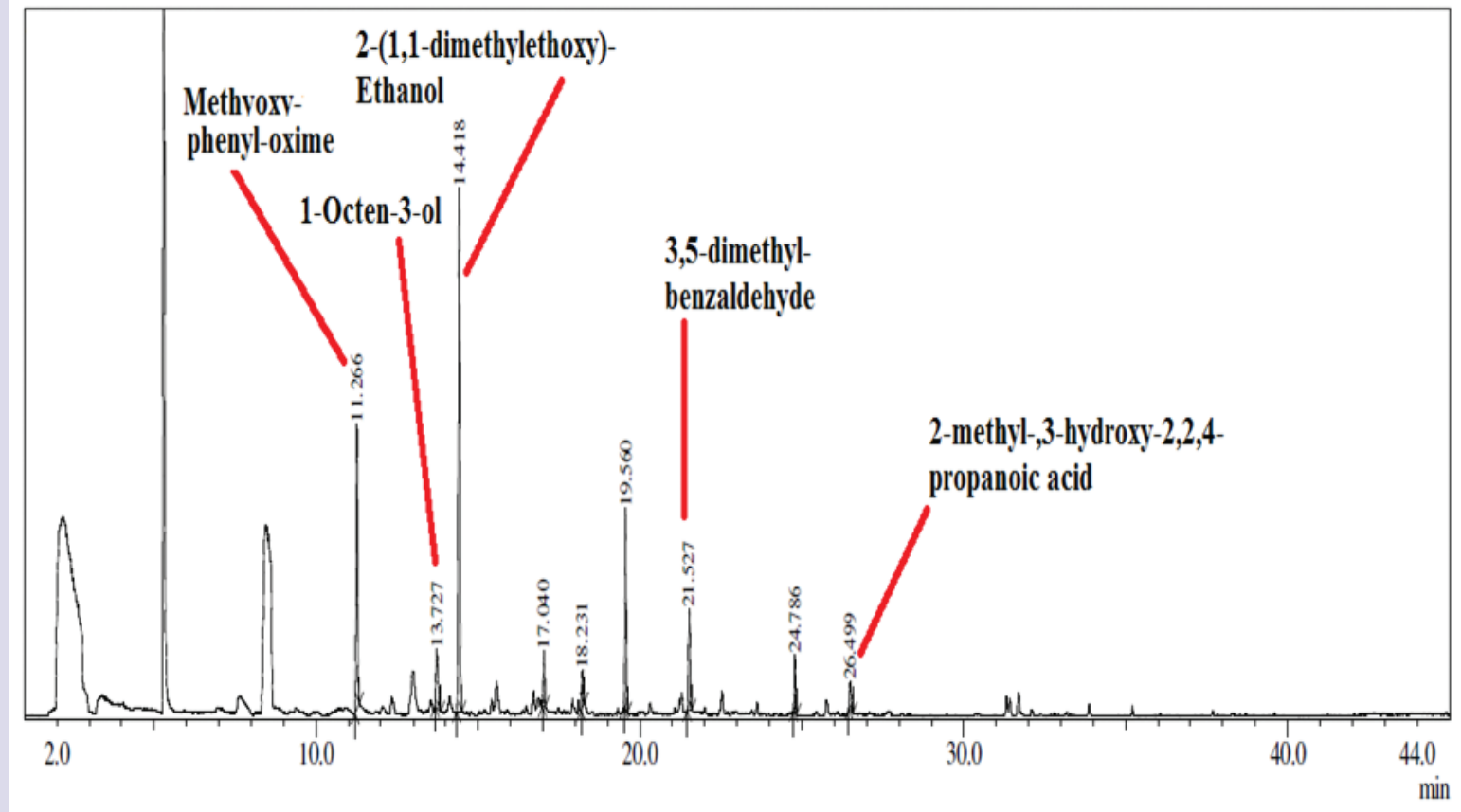

Figure 4: Gas chromatogram of a $0.5 \mu \mathrm{L}$ injection of $T$. ferdinandiana methanolic leaf extract. The extract was dried and resuspended in methanol for analysis. 


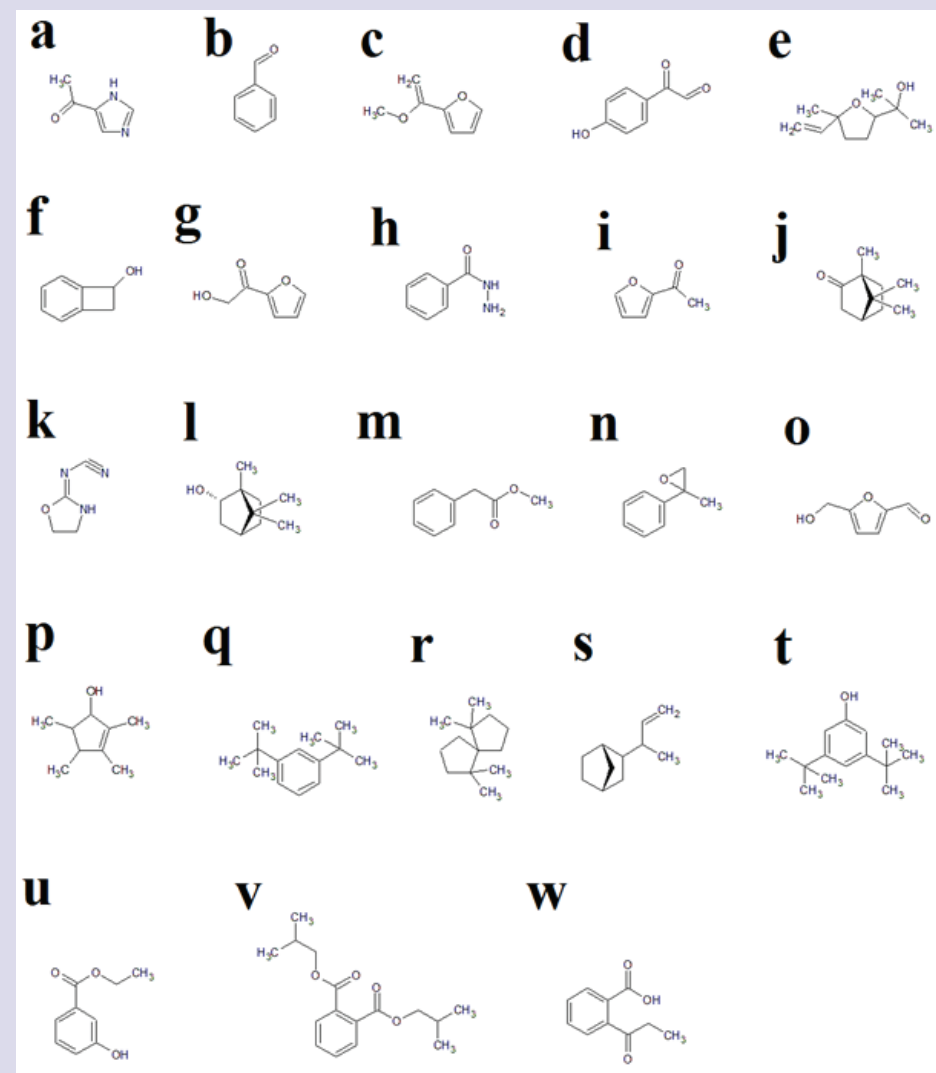

Figure 5: Chemical structures of T. ferdinandiana fruit methanolic extract compounds: (a) 1-(3H-imidazol-4-yl)-ethanone; (b) benzaldehyde; (c) methyl 2-furoate; (d) benzeneacetaldehyde; (e) linalool oxide; (f) bicyclo[4.2.0]octa-1,3,5-trien-7-ol; (g) furylhydroxymethyl ketone; (h) benzhydrazide; (i) 1-(2-furanyl)-ethanone; (j) camphor; (k) 2-(cyanoimino) oxazolidine; (I) borneal; (m) benzeneacetic acid, methyl ester; (n) 2-methyl-2-phenyl-oxirane; (o) 5-hydroxymethylfurfural; (p) 2,3,4,5-tetramethylcyclopent-2-en-1-ol; (q) 1,3-bis(1,1-dimethylethyl)-benzene; (r) 1,1,6,6-tetramethylspiro[4.4]nonane; (s) 2-(1-buten-3-yl)-bicyclo[2.2.1] heptane; (t) 3,5-bis(1,1-dimethylethyl)-phenol; (u) ethyl 3-hydroxybenzoate; (v) phthalic acid, diisobutylo ester; (w) 2-(1-oxopropyl)-benzoic acid.

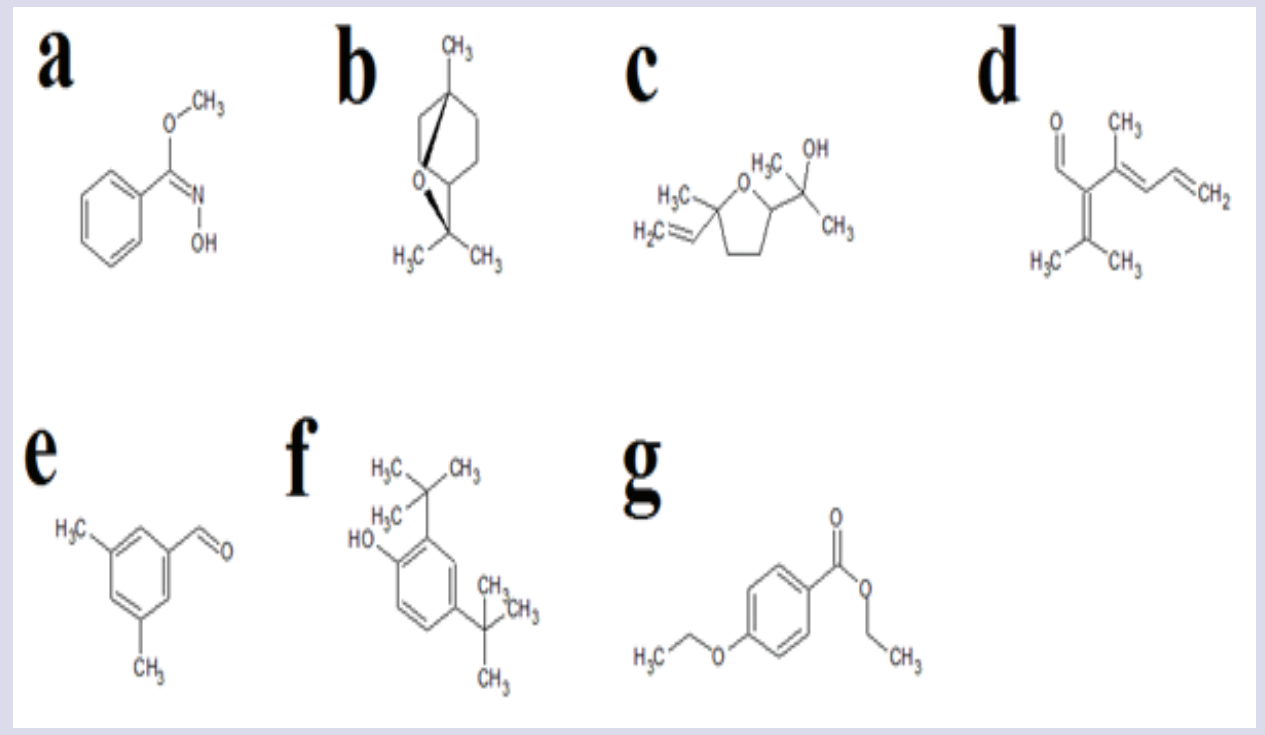

Figure 6: Chemical structures of T. ferdinandiana leaf methanolic extract compounds: (a) methyoxy-phenyl-oxime; (b) cineole; (c) linalool oxide; (d) 2-isopropylidene-3-methylhexa-3,5-dienal; (e) 3,5-dimethyl-benzaldehyde; (f) 2,4-di-tert-butylphenol; (g) ethyl p-ethoxybenzoate. 
(Figure 5h), benzeneacetic acid, methyl ester (Figure 5m), 2-methyl2-phenyl-oxirane (Figure 5n), 1,3-bis(1,1-dimethylethyl)-benzene (Figure 5q); 3,5-bis(1,1-dimethylethyl)-phenol (Figure 5t), ethyl 3-hydroxybenzoate (Figure $5 \mathrm{u}$ ), phthalic acid, diisobutylo ester (Figure $5 \mathrm{v}$ ) and 2-(1-oxopropyl)-benzoic acid (Figure $5 \mathrm{w}$ ) were also detected in the methanolic T. ferdinandiana fruit extract. Similarly, methyoxy-phenyloxime (Figure 6a), 3,5-dimethyl-benzaldehyde (Figure 6e),2,4-di-tertbutylphenol (Figure 6f) and ethyl p-ethoxybenzoate (Figure 6g) were putatively identified in the leaf methanolic extract. Several studies have previously reported potent antibacterial activity for phenolic benzaldehyde and benzoic acid derivatives. ${ }^{38}$ Thus, these compounds may also contribute to the $Y$. enterocolitica growth inhibitory activity reported in our study.

Several monoterpenoids were also putatively identified in the methanolic T. ferdinandiana extracts, with cis- and trans-linalool oxide (Figure 5e), camphor (Figure 5j) and borneol (Figure 5l) identified in the fruit extract. Linalool oxide was also present in the leaf methanolic extract, together with cineole (Figure 6b). Several other important terpenoids have also been reported in T. ferdinandiana extracts in recent studies using different analytical techniques. ${ }^{13}$ Monoterpenoids were particularly prevalent in those studies, with isomyocorene, cineole, cuminol, camphor andisomenthol reported in T. ferdinandiana fruit extracts. Many of these terpenoids have potent broad spectrum antibacterial activity ${ }^{39,42}$ and therefore may contribute to the Y. enterocolitica growth inhibitory activity. It is also likely that other phytochemical classes contribute to the growth inhibitory properties of these extracts. Our qualitative phytochemical screening studies indicate that polyphenolics, flavonoids, saponins, and terpenes were present in the T. ferdinandiana extracts. As our study used GC-MS techniques to putatively identify the phytochemical composition of the extracts, many of the mid to higher polarity compounds may have not been identified. Recent studies have reported the LC-MS profiles of similar T. ferdinandiana fruit ${ }^{11,14}$ and leaf extracts. ${ }^{16}$ Several features were common to all of these studies. All reported a diversity of tannins in both the fruit and leaf extracts. Gallic acid, ellagic acid and their methylated derivatives, chebulic acid, galloyl pyrogallol, corilagen, punicalin, castalagin and chebulagic acid were previously reported in similar T. ferdinandiana extracts to those tested in our study. These tannins have potent, broad spectrum growth inhibitory activity against a variety of bacterial species. ${ }^{40}$ Gallotannins have particularly well reported inhibitory properties. ${ }^{41}$ They function via multiple mechanisms including interacting with both cell surface proteins ${ }^{43,44}$ and through interactions with intracellular enzymes. ${ }^{45}$ Ellagitatannins also interact with cellular proteins and induce disruptions in bacterial cell walls. ${ }^{41,43}$

Recent studies also highlighted the stilbene components of T. ferdinandiana fruit extracts. ${ }^{13,14}$ Resveratrol and the glycosylated resveratrol derivative piceid, diethylstilbestrol monosulfate and combretastatin A1 were putatively identified in those studies. Identification of combretastatin A1 was particularly interesting as combretastatins have attracted much recent interest due to their potent ability to block cancer cell progression and induce apoptosis by binding intracellular tubulin, thereby disrupting microtubule formation. ${ }^{42}$ Whilst we were unable to find accounts of bacterial growth inhibition of combretastatin A1 in the literature, the growth inhibition of Staphylococcus aureus, Streptococcus pneumoniae, Escherichia coli and Neisseria gonorrhoeae by several synthetic combretastatins (and synthetic resveratrol analogues) have been reported. ${ }^{43}$ Therefore, it is likely that the T. ferdinandiana extract stilbene components may also contribute to the Y. enterocolitica growth inhibition noted in our study.

The findings reported here also indicate that the T. ferdinandiana fruit and leaf extracts were generally nontoxic, with $\mathrm{LC}_{50}$ values substantially $>1000 \mu \mathrm{g} / \mathrm{mL}$ in the Artemia nauplii bioassay. Indeed, only the
T. ferdinandiana leaf ethyl acetate extract had an $\mathrm{LC}_{50}$ value $<1000 \mu \mathrm{g} / \mathrm{mL}$. Whilst this extract inhibited $Y$. enterocolitica growth, the MIC value of $1100 \mu \mathrm{g} / \mathrm{mL}$ is indicative of moderate growth and thus has lower therapeutic potential in the treatment of yersiniosis compared to the methanolic extracts. Furthermore, an $\mathrm{LC}_{50}$ value of $767 \mu \mathrm{g} / \mathrm{mL}$ indicate low to moderate toxicity. However, this toxicity may impact on the usefulness of the leaf ethyl acetate extract as a medicinal antiseptic agent. Similar extracts prepared from fruits of these species have previously been reported to have high ascorbic acid levels. ${ }^{9}$ Whilst these ascorbic acid levels may have beneficial therapeutic effects, they may also be responsible (at least in part) for the toxicity reported here. Whilst A. franciscana have generally been reported to be a robust and hardy organism for toxicity screening, they are susceptible to $\mathrm{pH}$ changes. ${ }^{30}$ The levels of ascorbic acid previously reported in the $T$. ferdinandiana leaf ethyl acetateextract ${ }^{13,14}$ would be expected to have a significant impact on the $\mathrm{pH}$ of the seawater in the tests and this change may be responsible for the mortality induction reported in our study. Indeed, studies in our laboratory have shown that testing pure ascorbic acid in the concentrations previously reported to be in these extracts results in mortality similar to that reported here (unpublished results).

Toxicity was assessed in this study with the test organism A. franciscana. The lack of toxicity of these extracts indicates that they are likely to be safe for medicinal usage. This is hardly surprising as T. ferdinandiana is a food which is reputed to be highly nutritious. However, whilst the extracts examined in this report have potential as Y. enterocolitica growth inhibitory agents, caution is needed before these compounds can be applied to medicinal purposes. Toxicity towards A. franciscana has previously been shown to correlate well with toxicity towards human cells for many toxins. ${ }^{30}$ However, further studies are required to determine whether this is also true for the T. ferdinandiana extracts examined in these studies. The results of this study indicate that the T. ferdinandiana extracts examined in this study warrant further study due to their $Y$. enterocolitica growth inhibitory activity. Purification and identification of the bioactive components is needed to examine the mechanisms of action of these agents.

\section{CONCLUSION}

The results of this study demonstrate the potential of $T$. ferdinandiana fruit and leaf extracts to inhibit $Y$. enterocolitica growth. The methanolic T. ferdinandiana leaf and fruit extracts, as well as the fruit ethyl acetate extract, were particularly potent growth inhibitors, with MIC values generally $<350 \mu \mathrm{g} / \mathrm{mL}$. However, before being acceptable for therapeutic uses, further cell line toxicity studies are required to verify the safety of these extracts. Furthermore, studies aimed at the purification and identification of the bioactive components are required to examine the mechanisms of action of these extracts.

\section{ACKNOWLEDGEMENTS}

Financial support for this work was provided by the Environmental Futures Research Institute and the School of Natural Sciences, Griffith University, Australia. The authors are most grateful to David Boehme of Wild Harvest, Northern Territory, Australia for providing the T. ferdinandiana fruit pulp and leaves used in these studies.

\section{CONFLICTS OF INTEREST}

The authors report no conflicts of interest.

\section{ABBREVIATIONS USED}

DMSO: Dimethyl sulfoxide; $\mathbf{L C}_{50}$ : The concentration required to achieve $50 \%$ mortality; MIC: minimum inhibitory concentration. 


\section{REFERENCES}

1. Bancerz-Kisiel A, Shweda W. Yersiniosis-a zoonotic foodborne disease ofrelevance to public health. Annals of Agricultural and Environmental Medicine. 2015;22(3):397-402

2. Perry RD, Fetherston JD. Yersinia pestis-Etiologic Agent of Plague. Clinical Microbiology Reviews. 1997;10(1):35-66

3. Galindo CL, Rosenzweig JA, Kirtley ML. Pathogenesis of $Y$. enterocolitica and $Y$. pseudotuberculosis in Human Yersiniosis. Journal of Pathogens. 2011;(Vol issue missing):1-16.

4. Wright MH, Matthews B, Greene AC, et al. Growth inhibition of the zoonotic bacteria Bacillus anthracis by high antioxidant Australian plants: New leads for the prevention and treatment of anthrax. Pharmacognosy Communications. 2015;5(3):173-89.doi: 10.5530/pc.2015.3.3

5. Ong KL, Gould LH, Chen DL. Changing epidemiology of Yersinia enterocolitica infections: markedly decreased rates in young black children, Foodborne Diseases Active Surveillance Network (FoodNet), 1996-2009. Clinical Infectious Diseases. 2012;54(S5):S385-90. doi: 10.1093/cid/cis053

6. Bhaduri S, Wesley IV, Bush EJ. Prevalence of Pathogenic Yersinia enterocolitica Strains in Pigs in the United States. Applied and Environmental Microbiology. 2005; 71(11):7117-21.

7. Jiang GC, Kang DH, Fung DY. Enrichment procedures and plating media for isolation of Yersinia enterocolitica. Journal of Food Protection. 2000;63(11):1483-6.

8. Mohanty S, Cock IE. The chemotherapeutic potential of Terminalia ferdinandiana: Phytochemistry and bioactivity. Pharmacognosy Reviews. 2012;6(11):29-36. DOI: 10.4103/0973-7847.95855

9. Netzel M, Netzel G, Tian O. Native Australian fruits-a novel source of antioxidants for food. Innovative Food Science and Emerging Technologies. 2007;8(3):339-46.

10. Konczak I, Maillot F, Dalar A. Phytochemical divergence in 45 accessions of Ter minalia ferdinandiana (Kakadu plum). Food Chemistry. 2014;151 (issue missing): 248-56.

11. Rayan $P$, Matthews $B, M c D o n n e l l ~ P A$, et al . Terminalia ferdinandiana extracts as inhibitors of Giardia duodenalis proliferation: a new treatment for giardiasis. Parasitology Research. 2015;114(7):2611-20. DOI: 10.1007/s00436-015-4465-4

12. Courtney R, Sirdaarta J, Matthews B, et al. Tannin components and inhibitory activity of Kakadu plum leaf extracts against microbial triggers of autoimmune inflammatory diseases. Pharmacognosy Journal. 2015;7(1):18-31. DOI: 10.5530/ pj.2015.7.2

13. Sirdaarta J, Matthews B, White A, et al. GC-MS and LC-MS analysis of Kakadu plum fruit extracts displaying inhibitory activity against microbial triggers of multiple sclerosis. Pharmacognosy Communications. 2015;5(2):100-15. DOI: 10.5530/pc.2015.2.2

14. Sirdaarta J, Matthews B, Cock IE. Kakadu plum fruit extracts inhibit growth of the bacterial triggers of rheumatoid arthritis: Identification of stilbene and tannin components. Journal of Functional Foods. 2015;17:610-20. DOI: 10.1016/j.ff.2015.06.019

15. Cock IE, Mohanty S. Evaluation of the antibacterial activity and toxicity of Terminalia ferdinandiana fruit extracts. Pharmacognosy Journal. 2011;3(20):72-9.

16. Courtney R, Sirdaarta J, Matthews B, et al. Tannin components and inhibitory activity ofKakadu plum leaf extracts against microbialtriggers of autoimmune inflammatory diseases. Pharmacognosy Journal. 2015;7(1):18-31. DOI: 10.5530/ pj.2015.7.2

17. Kalt FR, Cock IE. Gas chromatography-mass spectroscopy analysis of bioactive Petalostigma extracts: Toxicity, antibacterial and antiviral activities. Pharmacognosy Magazine. 2014;10(37 Supp):S37-49. DOI: 10.4103/0973-1296.127338

18. Sautron C, Cock IE. Antimicrobial activity and toxicity of Syzygium australe and Syzygium leuhmannii fruit extracts. Pharmacognosy Communications. 2014;4(1):53-60. DOI: 10.5530/pc.2014.1.8

19. Vesoul J, Cock IE. The potential of Bunya nut extracts as antibacterial functional foods. Pharmacognosy Communications. 2012;2(1):72-9. DOI: 10.5530/ pc.2012.1.13

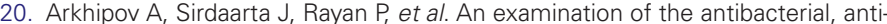
fungal, anti-Giardial and anticancer properties of Kigelia africana fruit extracts. Pharmacognosy Communications. 2014;4(3):62-76. DOI: 10.5530/pc.2014.3.7

21. Hart C, llanko P, Sirdaarta J, et al. Tasmanniastipitata as a functional food/natural preservative: Antimicrobial activity and toxicity. Pharmacognosy Communications. 2014;4(4):33-47. DOI: 10.5530/pc.2014.4.4

22. Jamieson N, Sirdaarta J, Cock IE. The anti-proliferative properties of Australian plants with high antioxidant capacities against cancer cell lines. Pharmacognosy
Communications. 2014;4(4):71-82. DOI: 10.5530/pc.2014.4.8

23. Cock IE, van Vuuren SF. Anti-Proteus activity of some South African medicinal plants: their potential for the prevention of rheumatoid arthritis. Inflammophar macology. 2014;22(1):23-36. DOI: 10.1007/s10787-013-0179-3

24. Winnett $\mathrm{V}$, Boyer $\mathrm{H}$, Sirdaarta J, et al. The potential of Tasmannia lanceolata as a natural preservative and medicinal agent: Antimicrobial activity and toxicity Pharmacognosy Communications. 2014;4(1):42-52. DOI: 10.5530/pc.2014.1.7

25. Kalt FR, Cock IE. The medicinal potential of Australian native plants from Toohey Forest, Australia. The South Pacific Journal of Natural Science. 2011;28(1):41-7.

26. Cock IE, van Vuuren SF. The potential of selected South African plants with antiKlebsiella activity for the treatment and prevention of ankylosing spondylitis Inflammopharmacology. 2014;23(1):21-35. DOI: 10.1007/s10787-014-0222-z

27. Cock IE. Antimicrobial activity of Acacia aulacocarpa and Acacia complanta methanolic extracts. Pharmacognosy Communications. 2012;2(1):66-71. DOI: 10.5530/pc.2012.1.12

28. Cock IE. Assessment of the toxicity of selected Australian native plant extracts using the Artemia franciscana nauplii bioassay. Internet Journal of Toxicology. 2008;5:2.

29. Ruebhart DR, Wikramasinghe WA, Cock IE. Protective efficacy of the antioxidants vitamin $\mathrm{E}$ and Trolox against Microcystis aeruginosa and microcystin-LR in Artemia franciscana nauplii. Journal of Toxicology and Environmental Health Part A. 2009;72(24):1567-75.

30. Cock IE, Ruebhart DR. Comparison of the brine shrimp nauplii bioassay and the ToxScreen-II test for the detection of toxicity associated with Aloe vera (Aloe barbadensis Miller) leaf extract. Pharmacognosy Research. 2009;1 (2):98-101.

31. Cock IE. The medicinal properties and phytochemistry of plants of the genus Terminalia (Combretaceae). Inflammopharmacology 2015;203-29. DOI 10.1007/ s10787-015-0246-z

32. Cock IE, Mohanty S. Evaluation of the antibacterial activity and toxicity of Terminalia ferdinandiana fruit extracts. Pharmacognosy Journal. 2011;3(20): 72-9. DOI: 10.5530/pj.2011.20.14

33. Wright $\mathrm{MH}$, Sirdaarta J, Matthews B, et al. Growth inhibitory activity of Kakadu Plum extracts against the opportunistic pathogen Clostridium perfringens: New leads in the prevention and treatment of clostridial myonecrosis. Pharmacognosy Journal. 2016:8(2):144-53. DOI: 10.5530/pj.2016.2.8

34. Ray B, Bhunia A. Fundamental Food Microbiology, $4^{\text {th }}$ edition, 2008; CRC Press, Boca Raton, USA.

35. Munoz-Davila MJ. Role of old antibiotics in the era of antibiotic resistance. Highlighted nitrofurantoin for the treatment of lower urinary tract infections. Antibiotics. 2014;3(1):39-48.

36. Che J, Zheng CJ, Song MX. Synthesis and antibacterial evaluation of furan derivatives bearing a rhodanine moiety. Medicinal Chemistry Research. 2014; 23(1):426-35.

37. Mital A. Synthetic nitroimidazoles: biological activities and mutagenicity relationships. Scientia Pharmaceutica. 2009;77(1):497-520.

38. Friedman M, Henika PR, Mandrell RE. Antibacterial activities of phenolic benzaldehydes and benzoic acids against Campylobacter jejuni, Escherichia coli, Listeria monocytogenes, and Salmonella enterica. Journal of Food Protection. 2003:66(1):1811-21.

39. Cock IE. The phytochemistry and chemotherapeutic potential of Tasmannialanceolata (Tasmanian pepper): A review. Pharmacognosy Communications. 2013;3(4):13-25.

40. Cock IE. The medicinal properties and phytochemistry of plants of the genus Terminalia (Combretaceae). Inflammopharmacology 2015; 203-29. DOI 10.1007/s10787-015-0246-z.

41. Buzzini $P$, Arapitsas $P$, Goretti M. Antimicrobial activity of hydrolysable tannins. Mini-Reviews Med Chem. 2008;8(12):1179-87.

42. Wolinsky LE, Sote EO. Isolation of natural plaque-inhibiting substances from Nigerian chewing sticks. Caries Res. 1984;18(3):216-25.

43. Hogg SD, Embery G. Blood-group-reactive glycoprotein from human saliva interacts with lipoteichoic acid on the surface of Streptococcus sanguiscells. Arch Oral Biol. 1982;27(3):261-8

44. Wu-Yuan CD, Chen CY, Wu RT. Gallotannins inhibit growth, watersolubleglucan synthesis, and aggregation of Streptococci mutans. J Dental Res. 1988;67(issue missing): 51-5.

45. Dark GG, Hill SA, Prise VE. Combretastatin A-4, an agent that displays potent and selective toxicity toward tumor vasculature. Cancer Res. 1997;57(10):1829-34 
PICTORIAL ABSTRACT

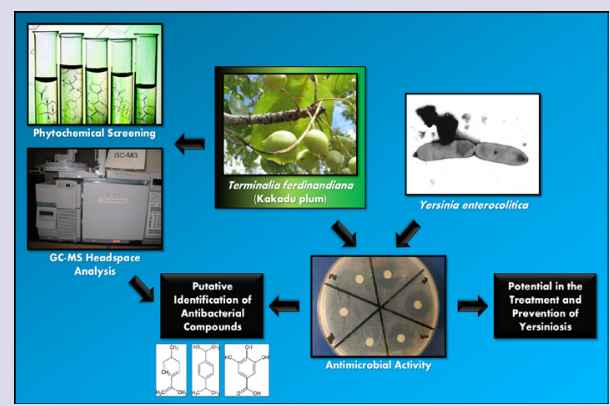

\section{SUMMARY}

- T. ferdinandiana fruit and leaf extracts were potent inhibitors of Yersinia enterocolitica growth.

- Methanolic leaf and fruit extracts were particularly potent, with MIC's of 372 and $123 \mu \mathrm{g} / \mathrm{mL}$ respectively.

- The ethyl acetate fruit extract was also a potent growth inhibitor (MIC $285 \mu \mathrm{g} / \mathrm{mL})$.

- All inhibitory extracts were either non-toxic or of low toxicity in the Artemia nauplii assay.

- GC-MS analysis identified several compounds that may contribute to the inhibitory activity.

\section{ABOUT AUTHORS}

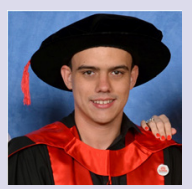

Dr Wright: Received his PhD in 2014, for his work investigating the manganese reduction and oxidation characteristics of environmental bacteria. He is currently a postdoctoral researcher at Griffith University, Australia, where he is working on several projects both in the areas of geomicrobiology and pharmacognosy. His present research interests are the use of bacteriogenic manganese oxides in the bioremediation of metal-contaminated sites as well as the use of Australian native plants in the treatment and prevention of various pathogenic bacteria.

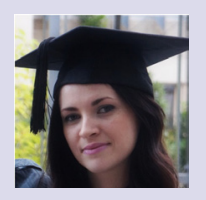

Megan Arnold: Is currently undertaking her PhD in Tropical Parasitology at Griffith University's Eskitis Institute for Drug Discovery with a focus on the identification and development of novel chemoprophylactic agents for malaria. Her other research interests include investigating Australian high antioxidant plants for their antibacterial capabilities.

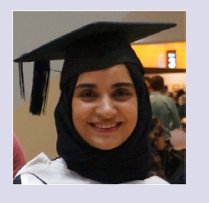

Ms. Huda Aldosary: Is undertaking her Ph.D. at Griffith University (Brisbane, Australia) under the supervision of Dr. Anthony Greene. Her primarily interests involve the investigation of bacterial hydrocarbon degradation and the potential for these processes in the remediation of contaminated sites. She has extensive experience with anaerobic organisms and specialises in extremophiles.

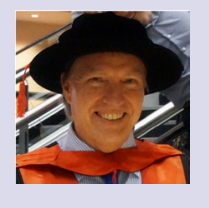

Dr Anthony Greene: Is a senior lecturer and researcher at Griffith University, Brisbane Australia. He obtained his PhD in Microbiology from the University of New South Wales and focuses on extreme environments, Bioremediation and Geomicrobiology. His specific interests include the microbial ecology of thermophilic, saline and alkaliphilic environments and the mechanisms and industrial potential of extremophilic bacteria contained therein.

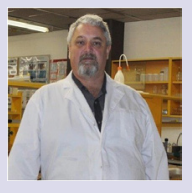

Dr lan Cock: Leads a research team in the Environmental Futures Research Institute and the School of Natural Sciences at Griffith University, Australia. His research involves bioactivity and phytochemical studies into a variety of plant species of both Australian and international origin, including Aloe vera, South Asian and South American tropical fruits, as well as Australia plants including Scaevola spinescens, Pittosporum phylliraeoides, Terminalia ferdinandiana (Kakadu plum), Australian Acacias, Syzygiums, Petalostigmas and Xanthorrhoea johnsonii (grass trees). This range of projects has resulted in nearly 200 publications in a variety of peer reviewed journals. 